\title{
ISOTROPIC RESOLUTION IN FLUORESCENCE IMAGING BY SINGLE PARTICLE RECONSTRUCTION
}

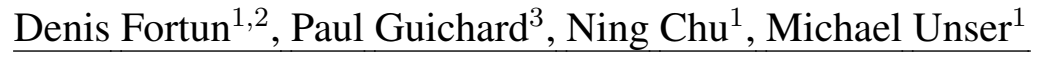 \\ ${ }^{1}$ Biomedical Imaging Group, EPFL, Switzerland \\ ${ }^{2}$ Center for Biomedical Imaging - Signal Processing core (CIBM-SP), EPFL, Switzerland \\ ${ }^{3}$ Department of Cell Biology, University of Geneva, Switzerland
}

\begin{abstract}
Low axial resolution is a major limitation of fluorescence imaging modalities. We propose a methodology to achieve high isotropic resolution by reconstructing fluorescence volumes from observations of multiple particle replicates with different orientations. The challenge is to conciliate high reconstruction accuracy, requiring a large amount of input 3D data, with computational tractability. We achieve this goal by designing an iterative joint deconvolution and multiview reconstruction algorithm with an efficient augmentedLagrangian based optimization. The computational cost is limited to only two FFTs per iterations, regardless of the number of input particles. We also adopt the nuclear norm of the Hessian as regularizer to avoid the usual staircase artifacts of the more standard total-variation. Experimental validation on realistic simulated data demonstrate the efficiency and accuracy of our method.
\end{abstract}

\section{INTRODUCTION}

Fluorescence microscopy is an essential tool for the observation of subcellular structures and the understanding of their interactions. A large variety of optical devices and reconstruction procedures have been proposed to enhance the resolution of fluorescence images. This research has particularly focused on the lateral plane, where confocal microscopes are able to reach the diffraction limit (around $200 \mathrm{~nm}$ ). This barrier is even surpassed by superresolution techniques [1, 2, 3]. However, no decisive advance has been realized to improve the axial resolution. As a consequence, super-resolution fluorescence imaging is still mainly restricted to the focal plane, which is likely to loose important 3D structure information.

To overcome this issue, we propose a novel conceptual framework that takes advantage of multiple poses to reconstruct fluorescence images with 3D isotropic high resolution. Our approach is to perform volumetric acquisitions of randomly oriented replicates of identical particles. This allows us

This work was supported in part by the European Research Council through the European Unions Seventh Framework Programme (FP7/20072013) under Grant 267439. to take advantage of the high lateral resolution of the microscope to gain access to details within each view. Our reconstruction then combines the details acquired at random poses.

This procedure is inspired by the single-particle reconstruction framework used in cryo electron microscopy (cryoEM) [4], where a particle is reconstructed from tomographic projections instead of volumetric acquisitions. To the best of our knowledge, the only similar existing work for fluorescence is the 3D averaging approach described in [5] for STORM. The selective-plane illumination microscopy technique (SPIM) acquires images of a single sample from multiple views and involves a related reconstruction problem, but it deals with a significantly lower number of views [6, 7]. Given an input volume, our overall framework is made of three steps: 1) detection of individual particles; 2) estimation of pose parameters; 3) 3D reconstruction. In this work, we demonstrate the feasibility and the potential of our approach by focusing on the reconstruction problem. The main challenge is to conciliate high reconstruction accuracy with computational efficiency. Indeed, while iterative methods with advanced regularization strategies are mandatory to achieve good results, the abundance of input 3D volumes is a major issue for computational tractability of this approach.

We propose a generic reconstruction framework to overcome the challenges mentioned above. We take the imaging modality into account by designing a joint approach that combines deconvolution and multiview reconstruction in a single model. We employ a second order regularizer based on the penalization of the nuclear norm of the Hessian, to favor piecewise-smooth solutions. To cope with the computational issue associated with the high number of views, we adopt a proximal splitting optimization strategy that we adapt to the specific structure of our data. The computational cost of each iteration is independent of the number of input orientations, which is the key point to be able to process large data. Altogether, this allows us to obtain accurate reconstructions with high axial resolution. We demonstrate the feasibility of our approach by extensive performance validation on realistic simulated data in confocal microscopy. Our work is a proof of concept and a first step toward the realization of a complete 
and practical framework that would include Steps 1) and 2). More details about our method can be found in a longer version of this paper [8].

\section{RECONSTRUCTION FROM MULTIPLE POSES}

\subsection{Model}

Consider a 3D micrograph containing a series of randomly oriented and shifted particle replicates $y_{\boldsymbol{\vartheta}_{i}}$, where $\boldsymbol{\vartheta}_{i} \in \mathbb{R}^{6}$ is a vector of pose parameters composed by rotation parameters $\boldsymbol{\theta}_{i} \in \mathbb{R}^{3}$ and translation parameters $\mathbf{t}_{i} \in \mathbb{R}^{3}$. Let us assume that the particles have been detected and the pose parameters are known. The goal is to reconstruct the model $x$ of the particle with high isotropic resolution.

We consider the case of confocal imaging, for which the image-acquisition process can be modeled as the convolution of the underlying signal with a PSF denoted by $h$. Thus, we have at pixel $\mathbf{p}$ in the image domain

$$
y_{\boldsymbol{\vartheta}_{i}}(\mathbf{p})=\int_{\mathbb{R}^{3}} h(\mathbf{q}) x\left(\mathbf{R}_{\boldsymbol{\theta}_{i}}(\mathbf{p}-\mathbf{q})+\mathbf{t}_{i}\right) \mathrm{d} \mathbf{q},
$$

where $\mathbf{R}_{\boldsymbol{\theta}_{i}} \in \mathbb{R}^{3 \times 3}$ is the rotation matrix with angular parameters $\boldsymbol{\theta}_{i}$. After alignment of the observed particles, (1) can be rewritten, with changes of variables:

$$
y_{\boldsymbol{\vartheta}_{i}}\left(\mathbf{R}_{\boldsymbol{\theta}_{i}}^{-1}\left(\mathbf{p}-\mathbf{t}_{\mathbf{i}}\right)\right)=\int_{\mathbb{R}^{3}} h\left(\mathbf{R}_{\boldsymbol{\theta}_{i}}^{-1} \mathbf{q}\right) x(\mathbf{p}-\mathbf{q}) \mathrm{d} \mathbf{q} .
$$

In a discrete setting, assuming that all images contain $M$ pixels, we denote $\mathbf{y}_{i} \in \mathbb{R}^{M}$ the observation vector corresponding to the discretization of $y_{\boldsymbol{\vartheta}_{i}}\left(\mathbf{R}_{\boldsymbol{\theta}_{i}}^{-1}\left(\cdot-\mathbf{t}_{\mathbf{i}}\right)\right), \mathbf{H}_{\boldsymbol{\vartheta}_{i}} \in \mathbb{R}^{M \times M}$ the convolution matrix of $h\left(\mathbf{R}_{\boldsymbol{\theta}_{i}}^{-1} \cdot\right)$, and $\mathbf{x} \in \mathbb{R}^{M}$ the unknown image in vector form. The discretization of (2) leads to

$$
\mathbf{y}_{i}=\mathbf{H}_{\vartheta_{i}} \mathbf{x}
$$

\subsection{Reconstruction problem}

We formulate the reconstruction problem as the following maximum a posteriori (MAP) estimation:

$$
\widehat{\mathbf{x}}=\underset{\mathbf{x} \in \mathbb{R}_{+}^{M}}{\arg \min } \frac{\lambda}{2} \sum_{i=1}^{N}\left\|\mathbf{y}_{i}-\mathbf{H}_{\boldsymbol{\vartheta}_{i}} \mathbf{x}\right\|_{2}^{2}+\phi(\mathbf{x}),
$$

where $\phi(\mathbf{x})$ is a regularization term, $N$ is the number of particles, and $\lambda>0$ is a tradeoff parameter. The quadratic penalization in the data term reflects the assumption of additive Gaussian noise, and the solution is imposed to belong to $\mathbb{R}_{+}^{M}$.

We adopt a second order regularization strategy involving the discrete Hessian operator $\mathcal{H}: \mathbb{R}^{M} \rightarrow \mathbb{R}^{M \times 3 \times 3}$ as defined in [9]. The nuclear norm of a matrix $\mathbf{X}$ is defined as $\|\mathbf{X}\|_{N}=\sum_{i=1}^{K}\left|\sigma_{i}\right|$, where $\sigma_{i}$ is the $i^{t h}$ singular value of $\mathbf{X}$. Our regularizer is then given by

$$
\phi(\mathbf{x})=\|\mathcal{H} \mathbf{x}\|_{1, N}=\sum_{i=1}^{M}\left\|[\mathcal{H} \mathbf{x}]_{i}\right\|_{N}
$$

Singular values of the Hessian can be interpreted as local measures of curvature, and $\ell_{1}$ penalization promotes piecewise-smooth (opposed to piecewise-constant) solutions.

\subsection{Minimization}

The main issue in the optimization stage is to be able to handle a large amount of 3D observations at a reasonable computational cost. Our solution involves the decomposition of the original problem into efficiently solvable sub-problems, via the alternating-direction method of multipliers (ADMM) $[10,11]$, which has become very popular in the field. We first reformulate (4) as a constrained optimization problem by introducing splitting variables $\mathbf{u}_{1}$ and $\mathbf{u}_{2}$, respectively associated to the regularization term and the positivity constraint:

$$
\min _{\mathbf{x} \in \mathbb{R}^{M}} \frac{\lambda}{2} \sum_{i=1}^{N}\left\|\mathbf{y}_{i}-\mathbf{H}_{\boldsymbol{\vartheta}_{i}} \mathbf{x}\right\|_{2}^{2}+g(\mathbf{u}), \text { s.t. } \mathbf{u}=\mathbf{A} \mathbf{x}
$$

where $\mathbf{u}=\left[\mathbf{u}_{1}, \mathbf{u}_{2}\right]^{\top}, \mathbf{A}=[\mathcal{H}, \mathbf{I}]^{\top}$ with $\mathbf{I}$ the identity matrix, and $g(\mathbf{u})=\left\|\mathbf{u}_{1}\right\|_{1, p}+\iota_{\mathbb{R}_{+}^{M}}\left(\mathbf{u}_{2}\right)$ whit $\iota_{\mathbb{R}_{+}^{M}}$ the indicator function of $\mathbb{R}_{+}^{M}$. The augmented-Lagrangian formulation of the problem (6) is then

$$
\begin{aligned}
L_{\mu}(\mathbf{x}, \mathbf{u}, \boldsymbol{\alpha})=\frac{\lambda}{2} \sum_{i=1}^{N} & \left\|\mathbf{y}_{i}-\mathbf{H}_{\boldsymbol{\vartheta}_{i}} \mathbf{x}\right\|_{2}^{2}+g(\mathbf{u}) \\
& +\boldsymbol{\alpha}^{\top}(\mathbf{u}-\mathbf{A x})+\frac{\mu}{2}\|\mathbf{u}-\mathbf{A x}\|_{2}^{2}
\end{aligned}
$$

where $\boldsymbol{\alpha}=\left[\boldsymbol{\alpha}_{1}, \boldsymbol{\alpha}_{2}\right]^{\top}$ is the Lagrange multiplier with $\boldsymbol{\alpha}_{1} \in$ $\mathbb{R}^{3 M}, \boldsymbol{\alpha}_{2} \in \mathbb{R}^{M}$, and $\mu$ is the parameter associated to the quadratic penalty. Problem (4) is then solved by the following alternated updates of the variables:

$$
\begin{aligned}
& \mathbf{x}^{k+1}=\underset{\mathbf{x} \in \mathbb{R}^{M}}{\arg \min } L_{\mu}\left(\mathbf{x}, \mathbf{u}^{k}, \boldsymbol{\alpha}^{k}\right) \\
& \mathbf{u}^{k+1}=\underset{\mathbf{u} \in \mathbb{R}^{4 M}}{\arg \min } L_{\mu}\left(\mathbf{x}^{k+1}, \mathbf{u}, \boldsymbol{\alpha}^{k}\right) \\
& \boldsymbol{\alpha}^{k+1}=\boldsymbol{\alpha}^{k}+\mu\left(\mathbf{A} \mathbf{x}^{k+1}-\mathbf{u}^{k+1}\right) .
\end{aligned}
$$

The two components of $\mathbf{u}$ are independent in the subproblem (9), so that the minimization w.r.t. the two variables can be performed separately. The update of $\mathbf{u}_{1}$ amounts to the computation of pixel-wise proximal operators of the nuclear norm. It can be efficiently computed by singular value thresholding [9]. The update of $\mathbf{u}_{2}$ is the projection onto the set $\mathbb{R}_{+}^{M}$, $\mathbf{u}_{2}^{k+1}=\max \left(\mathbf{v}_{2}^{k}, 0\right)$, with $\mathbf{v}_{2}^{k}=\mathbf{x}^{k+1}-(1 / \mu) \boldsymbol{\alpha}_{2}^{k}$.

The subproblem (8) is a pixelwise quadratic problem solvable in closed form as

$$
\begin{aligned}
\mathbf{x}^{k+1}= & \left(\mu \mathbf{A}^{\top} \mathbf{A}+\lambda \sum_{i=1}^{N} \mathbf{H}_{\boldsymbol{\vartheta}_{i}}^{\top} \mathbf{H}_{\boldsymbol{\vartheta}_{i}}\right)^{-1} \\
& \left(\mu \mathbf{A}^{\top}\left(\mathbf{u}^{k}-\frac{\boldsymbol{\alpha}^{k}}{\mu}\right)+\lambda \sum_{i=1}^{N} \mathbf{H}_{\boldsymbol{\vartheta}_{i}}^{\top} \mathbf{y}_{i}\right) .
\end{aligned}
$$


Table 1. Comparison of PSNR (dB) of the reconstructions of our method with TV and HN regularizations, Deconv/Average, and Average [5], for 100 orientation angles and several levels of noise.

\begin{tabular}{|c|ccc|ccc|}
\hline Volume & \multicolumn{3}{|c|}{ Centriole [12] } & \multicolumn{3}{c|}{ Nuclear pore [13] } \\
Noise variance & 1 & 5 & 10 & 1 & 5 & 10 \\
\hline Average [5] & 10.84 & 10.63 & 10.50 & 8.55 & 8.33 & 8.32 \\
Deconv/Average & 19.80 & 18.00 & 17.71 & 20.28 & 19.66 & 18.57 \\
Proposed-TV & 23.14 & 20.22 & 20.01 & 23.72 & 21.28 & 20.84 \\
Proposed-HN & $\mathbf{2 3 . 7 9}$ & $\mathbf{2 1 . 1 0}$ & $\mathbf{2 0 . 1 7}$ & $\mathbf{2 4 . 0 5}$ & $\mathbf{2 1 . 8 3}$ & $\mathbf{2 0 . 9 2}$ \\
\hline
\end{tabular}

Most of the computational cost of the algorithm is devoted to the matrix multiplications by $\mathbf{H}_{\vartheta_{i}}^{\top}$ in (11), summed over a large number $N$ of particles. The crucial advantage of the ADMM approach is to decouple these operations from the iterative updates. The sums can then be computed offline, which makes the cost of each iteration independent of the number of orientations.

Another computational issue comes from the fact that the matrix $\left(\mu \mathbf{A}^{\top} \mathbf{A}+\lambda \sum_{i=1}^{N} \mathbf{H}_{\boldsymbol{\vartheta}_{i}}^{\top} \mathbf{H}_{\boldsymbol{\vartheta}_{i}}\right)$ is too large to be inverted. Therefore, we perform the update (11) efficiently in the Fourier domain after diagonalization of the blockcirculant the matrices $\mathbf{H}_{\vartheta_{i}}$ and $\mathbf{D}$, which leads to

$\mathbf{x}^{k+1}=\mathcal{F}^{-1}\left\{\frac{\mathcal{F}\left\{\mu \mathbf{A}^{\top}\left(\mathbf{u}^{k}-\frac{\boldsymbol{\alpha}^{k}}{\mu}\right)\right\}+\lambda \sum_{i=1}^{N} \mathcal{F}\left\{\mathbf{H}_{\boldsymbol{\vartheta}_{i}}^{\top} \mathbf{y}_{i}\right\}}{\mu\left(|\mathcal{F}\{\mathbf{D}\}|^{2}+\mathbf{I}\right)+\lambda \sum_{i=1}^{N}\left|\mathcal{F}\left\{\mathbf{H}_{\boldsymbol{\vartheta}_{i}}\right\}\right|^{2}}\right\}$

The terms $\sum_{i=1}^{N} \mathbf{H}_{\boldsymbol{\vartheta}_{i}}^{\top} \mathbf{y}_{i}$ and $\mu\left(|\mathcal{F}\{\mathbf{D}\}|^{2}+\mathbf{I}\right)+\lambda \sum_{i=1}^{N}\left|\mathcal{F}\left\{\mathbf{H}_{\boldsymbol{\vartheta}_{i}}\right\}\right|^{2}$ are constant and computed offline. The computational time of each iteration is then mostly devoted to two FFTs, and the total number of FFT computations to reconstruct a particle is $2\left(N+n_{\text {it }}+1\right)$, where $n_{\text {it }}$ is the number of iterations of the ADMM optimization. The time complexity of the algorithm is thus $\mathcal{O}(N \log (N))$, whereas independent deconvolutions of each particle would require $O\left(N^{2} \log (N)\right)$.

\section{RESULTS}

To evaluate the performance of our method, we simulate input particles according to the forward model (3) from CryoEM reconstructions available in the database EMDataBank ${ }^{1}$ (see Figure 1a,b,c). In regard of their very high (subnanometric) resolution, Cryo-EM reconstructions can be used as ground truth for fluorescence imaging. We selected the centriole basal body [12] and the nuclear pore [13], which are large enough to be imaged by a confocal microscope. We compare our results with those of the only existing competing method [5], which reconstructs by averaging the input particles. We also extend the method of [5] by introducing a first step of independent deconvolutions with total-variation regularization (TV) of each particle before averaging. We refer to this extension as Deconv/Average. To evaluate the impact of the

\footnotetext{
${ }^{1}$ http://www.emdatabank.org/search.html
}

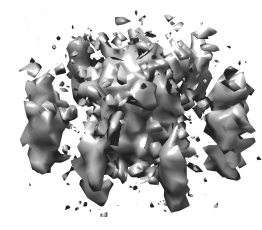

(a) Input particle 1

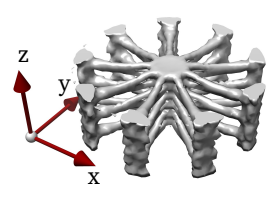

(c) Ground truth

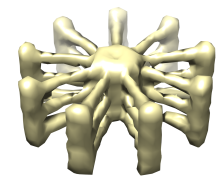

(e) Proposed-TV
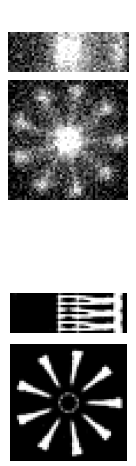

(b) Input particle 2

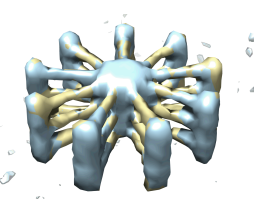

(d) Proposed-TV + Deconv/Average
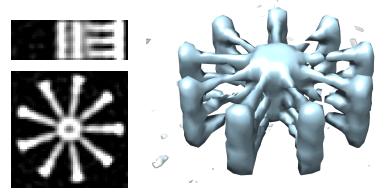

(f) Deconv/Average
Fig. 1. Visual comparisons of reconstruction results with 100 angles and noise variance of $\sigma^{2}=5$, in the case of the centriole[12].

Hessian nuclear norm regularization (denoted Proposed- $H N$ ), we compare it with the TV approach (denoted Proposed-TV). In our experiments, the PSF are generated according to the Born\&Wolf model with the available PSF Generator software ${ }_{2}^{2}$ described in [14]. The PSF support in pixels is $(69 \times 69 \times 43)$ and the input volume size is $(70 \times 70 \times 35)$.

The PSNR results reported in Table 1 for 100 angles and various levels of noise demonstrate the higher accuracy yielded by our joint approach in all the cases. Visual results in Figure 1 highlights the better preservation of structure details by our method, and the isotropic resolution of the results. The superiority of the Hessian nuclear norm regularization over TV can also be clearly observed in Table 1 .

The influence of the number of poses on the PSNR of the reconstructions is reported in Figure 2 for our method and $D e$ conv/Average. Increasing the number of poses improves the accuracy of both methods. However, the PSNR of our method grows significantly faster than the one of Deconv/Average. This is a very important point in practice because it allows us to reduce the required number of particles, which can facilitate the preparation of samples for the biologists.

We observe in Figure 3 that the computational time of our method stays almost constant as the number of poses increases, whereas it grows much faster in the case of $D e$ conv/Average. The reason is that the iterative part of our method, which contains most of the computational cost, does not depend on the number of poses. For 100 poses, our method takes $30 \mathrm{~s}$ and Deconv/Average takes $29 \mathrm{~min}$. This is a key result since in practice the reconstruction needs to be performed several times, alternatively with angles estimation, which makes the Deconv/Average approach prohibitively 


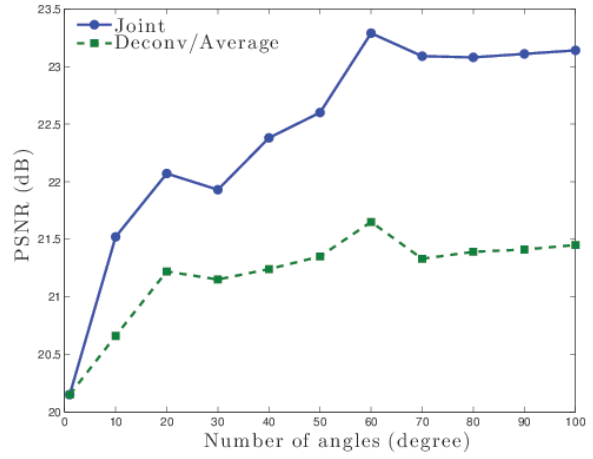

Fig. 2. Influence of the number of orientations on our method and Deconv/Average in the case of the centriole [12].

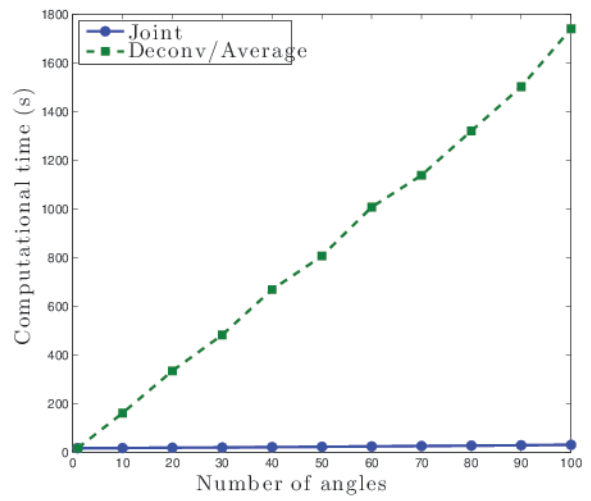

Fig. 3. Impact of the number of orientations on the computational time of our method and Deconv/Average in the centriole case [12].

slow in practice. The feasibility of the reconstruction is thus established by the low computational cost of our method.

\section{CONCLUSION}

We have proposed a novel approach for reconstructing fluorescence volumes with isotropic high resolution. We exploit multiple poses of particle replicates to combine multiview reconstruction and deconvolution in the same model. The computational cost of the iterative part of our optimization approach does not depend on the number of input volumes. This allows us to tackle the challenge of processing a large amount of 3D data in low computational time. Experimental results demonstrate the feasibility of our scheme by achieving accurate reconstructions in realistic situations, and outperforming similar approaches based on averaging in all situations.

\section{REFERENCES}

[1] M.G.L. Gustafsson, "Surpassing the lateral resolution limit by a factor of two using structured illumination microscopy," Journal of Microscopy, vol. 198, no. 2, pp. 82-87, 2000.

[2] H.W. and J. Wichmann, "Breaking the diffraction resolution limit by stimulated emission: stimulated-emission-depletion fluorescence microscopy," Optics Letters, vol. 19, no. 11, pp. 780-782, 1994.

[3] E. Betzig, G.H. Patterson, R. Sougrat, O.W. Lindwasser, S. Olenych, J.S. Bonifacino, M.W. Davidson, J. LippincottSchwartz, and H.F. Hess, "Imaging intracellular fluorescent proteins at nanometer resolution," Science, vol. 313, no. 5793, pp. 1642-1645, 2006.

[4] S.H.W. Scheres, R. Núñez-Ramírez, C.Ò.S. Sorzano, J.M. Carazo, and R. Marabini, "Image processing for electron microscopy single-particle analysis using Xmipp," Nature Protocols, vol. 3, no. 6, pp. 977-990, 2008.

[5] J. Broeken, H. Johnson, D.S. Lidke, S. Liu, R.P.J. Nieuwenhuizen, S. Stallinga, K.A. Lidke, and B. Rieger, "Resolution improvement by 3D particle averaging in localization microscopy," Methods and Applications in Fluorescence, vol. 3, no. 1, pp. $014003,2015$.

[6] M. Temerinac-Ott, O. Ronneberger, P. Ochs, W. Driever, T. Brox, and H. Burkhardt, "Multiview deblurring for 3-d images from light-sheet-based fluorescence microscopy," IEEE Transactions on Image Processing, vol. 21, no. 4, pp. 18631873, 2012.

[7] S. Preibisch, F. Amat, E. Stamataki, M. Sarov, R.H. Singer, E. Myers, and P. Tomancak, "Efficient Bayesian-based multiview deconvolution," Nature Methods, vol. 11, no. 6, pp. 645-648, 2014.

[8] D. Fortun, P. Guichard, N. Chu, and M. Unser, "Reconstruction from multiple poses in fluorescence imaging: proof of concept," IEEE Journal of Selected Topics in Signal Processing, in press, 2016.

[9] S. Lefkimmiatis, J.P. Ward, and M. Unser, "Hessian Schattennorm regularization for linear inverse problems," IEEE Transactions on Image Processing, vol. 22, no. 5, pp. $\overline{1873-}$ 1888, May 2013.

[10] Y. Wang, J. Yang, W. Yin, and Y. Zhang, "A new alternating minimization algorithm for total variation image reconstruction," SIAM Journal on Imaging Sciences, vol. 1, no. 3, pp. 248-272, 2008.

[11] S. Ramani and J.A. Fessler, "A splitting-based iterative algorithm for accelerated statistical x-ray CT reconstruction," IEEE Transactions on Medical Imaging, vol. 31, no. 3, pp. 677-688, 2012.

[12] P. Guichard, V. Hachet, N. Majubu, A. Neves, D Demurtas, N. Olieric, I. Fluckiger, A. Yamada, K. Kihara, Y. Nishida, S. Moriya, M.O. Steinmetz, Y. Hongoh, and P. Gönczy, "Native architecture of the centriole proximal region reveals features underlying its 9-fold radial symmetry," Current Biology, vol. 23, no. 17, pp. 1620-1628, 2013.

[13] M. Beck, F. Förster, M. Ecke, J.M. Plitzko, F. Melchior, G. Gerisch, W. Baumeister, and O. Medalia, "Nuclear pore complex structure and dynamics revealed by cryoelectron tomography," Science, vol. 306, no. 5700, pp. 1387-1390, 2004.

[14] H. Kirshner, F. Aguet, D. Sage, and M. Unser, “3-D PSF fitting for fluorescence microscopy: Implementation and localization application," Journal of Microscopy, vol. 249, no. 1, pp. 13-25, 2013. 